\title{
El Comportamiento organizacional frente a situaciones de cambio. ¿Cómo enfrentarlos?
}

Organizational behavior in situations of change. How to deal with them?

1 Sandra Janeth Paredes Jiménez Universidad Estatal de Bolívar, Guanujo, Ecuador sparedes@ueb.edu.ec

2 Gabriela Paola Carrasco Lara Universidad Estatal de Bolívar gcarrasco@ueb.edu.ev

3 Paola Estefania Albán Trujillo Universidad Estatal de Bolívar, Guanujo Ecuador palban@ueb.edu.ec
(iD) https://orcid.org/0000-0003-1689-1744

(iD) https://orcid.org/0000-0002-4635-1418

https://orcid.org/0000-0001-8311-5767

Artículo de Investigación Científica y Tecnológica

Enviado: 06/12/2021

Revisado: $21 / 12 / 2021$

Aceptado: 28/01/2022

Publicado:05/02/2022

DOI: https://doi.org/10.33262/ap.v4i1.1.138

Cítese:

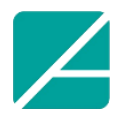

Ciencia

Digital

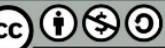

Paredes Jiménez, S. J., Carrasco Lara, G. P., \& Albán Trujillo, P. E. (2022). El Comportamiento organizacional frente a situaciones de cambio. ¿Cómo enfrentarlos?. AlfaPublicaciones, 4(1.1), 30-41. https://doi.org/10.33262/ap.v4i1.1.138

ALFA PUBLICACIONES, es una Revista Multidisciplinar, Trimestral, que se publicará en soporte electrónico tiene como misión contribuir a la formación de profesionales competentes con visión humanística y crítica que sean capaces de exponer sus resultados investigativos y científicos en la misma medida que se promueva mediante su intervención cambios positivos en la sociedad. https://alfapublicaciones.com

La revista es editada por la Editorial Ciencia Digital (Editorial de prestigio registrada en la Cámara Ecuatoriana de Libro con No de Afiliación 663) www.celibro.org.ec

Esta revista está protegida bajo una licencia Creative Commons Attribution Non Commercial No Derivatives 4.0 International. Copia de la licencia: $\underline{\text { http://creativecommons.org/licenses/by-nc-nd/4.0/ }}$ 


\section{Palabras claves:} comportamiento, evolución, cambios, aprendizaje.

\section{Keywords:}

behavior, evolution, changes, learning.

\section{Resumen}

El objetivo general de esta investigación se basa en analizar el comportamiento organizacional frente a situaciones de cambio y como son capaces los trabajadores de enfrentarlos y poder mantener sus actividades laborales en orden. La metodología utilizada tiene un diseño bibliográfico de tipo documental. Se establecen conceptos que permiten mostrar el comportamiento organizacional como una herramienta que sirve para conocer la interacción entre los individuos dentro de una organización y como son capaces de enfrentare los cambios que surgen durante la evolución propia de la empresa. Como conclusión se menciona que los cambios son parte de la evolución de las organizaciones, por lo que la preparación, el aprendizaje y el mejoramiento profesional son herramientas que sirven para cubrir las necesidades que se tengan en las áreas de trabajo, y sean capaces de atravesar estos cambios de forma positiva, que sean sinónimo de progreso y beneficios para todos. Las organizaciones deben tener muy claras las metas y objetivos trazados, con el propósito de definir estrategias que se adecúen para el logro de estas, así como también conocer las conductas de cada uno de sus trabajadores, de manera que la estructura organizacional no se vea afectada por posibles cambios que puedan surgir dentro de la empresa.

\section{Abstract}

The general objective of this research is based on analyzing organizational behavior in the face of change situations and how workers can cope with them and be able to keep their work activities in order. The methodology used has a bibliographic design of a documentary type. Concepts are established that allow showing organizational behavior as a tool that serves to know the interaction between individuals within an organization and how they can face the changes that arise during the evolution of the company itself. As a conclusion it is mentioned that changes are part of the evolution of organizations, so that preparation, learning and professional improvement are tools that serve to meet the needs that are had in the work areas and can go through these changes in a positive way, that are synonymous with progress and benefits for all. Organizations must be very clear about the goals and objectives set, with the purpose of 
defining strategies that are appropriate for their achievement, as well as knowing the behaviors of each of their workers, so that the organizational structure is not seen affected by possible changes that may arise within the company.

\section{Introducción}

Las actividades alrededor del mundo siempre están en constante movimiento, lo que genera en las personas diversos cambios que los lleva a adaptarse a estos, buscando la mejor manera de afrontar las situaciones que se presentan a lo largo de sus vidas. Esto mismo pasa en los lugares de trabajo, que por diversas razones sufren cambios, bien sea por movimientos en la estructura organizacional, cambios financieros o de cualquier índole que genere en los implicados la capacidad de adaptarse a estas situaciones.

Hoy en día las organizaciones están siempre en la búsqueda de personal que sea capaz de demostrar sus habilidades para ser productivos y que los conocimientos que estos posean sean suficientes para afrontar situaciones nuevas y fortuitas, y que estos cambios no afecten de manera negativa la cultura y el comportamiento organizacional de la empresa, y que el engagement en su trabajo diario no se pierda mientras enfrentan nuevos retos.

En palabras de Armijo y Portalanza (2017) el engagement es definido como un estado de vigor, dedicación y absorción en un trabajador de una empresa. En este sentido, el engagement se evidencia en las empresas por medio de los resultados que refleja la organización y aquellos trabajadores que demuestren mayor engagement son los que perduraran en la empresa creciendo y fortaleciéndose a nivel profesional dentro de la misma que a su vez se convierte en satisfacción laboral.

Existe una gran diversidad de personalidades dentro del mundo laboral, lo que hace más complejo el análisis de los perfiles laborales, y la formación de grupos de trabajo que sean capaces de adaptarse y demostrar sus habilidades en su área de trabajo.

Por tanto, las organizaciones, están siempre en la búsqueda de estrategias que permitan formar ambientes de trabajo adecuados, agradables y propicios para el desarrollo de las actividades propias de la naturaleza de la empresa, que faciliten el trabajo y la comunicación organizacional (Molina-Germán et al., 2019).

Estos aspectos antes mencionados se refieren a la cultura organizacional, tema que ha sido estudiado a lo largo de los años por diversos autores donde mencionan que esta, es un conjunto de conocimientos compartidos entre los miembros de un determinado grupo que a través de las actividades diarias compartidas adquieren experiencias. De la misma 
manera, se menciona que estas experiencias les ayudan a adquirir hábitos, valores y conductas que se extiende a nuevos empleados y que no solo son aplicadas dentro de su área laboral, sino que trasciende a la sociedad (Molina-Germán et al., 2019)

Estos aspectos también van de la mano con el compromiso laboral que pueda tener el trabajador con su puesto de trabajo y con la empresa el cual según Félix et al. (2018), es un factor determinante para que la persona permanezca en su puesto de trabajo y sea capaz de enfrentar cambios tanto positivos como negativos que puedan afectar el ambiente laboral.

De esta manera, en esta investigación se pretende analizar el comportamiento organizacional frente a situaciones de cambio y como son capaces los trabajadores de enfrentarlos y poder mantener sus actividades laborales en orden. La metodología utilizada tiene un diseño bibliográfico de tipo documental.

\section{Metodología}

Esta investigación se realiza a través de una metodología documental, basada en la revisión de artículos de diferentes autores, indica Grajales (2000), la investigación documental es aquella que se realiza a través de la consulta de documentos (libros, revistas, periódicos, memorias, anuarios, registros, códices, constituciones, etc.). En ese orden de ideas, en este proceso de búsqueda, recuperación, análisis, e interpretación de datos registrados por otros investigadores en fuentes documentales, permite poder dilucidar ideas que logren establecer puntos de vista que sirvan como base para otras investigaciones.

\section{Resultados y Discusión}

\section{Comportamiento Organizacional}

Este inició en los años 60, dentro de las ciencias de la administración de empresas y con el paso del tiempo los diversos autores que han estudiado esta área del conocimiento se han apoyado en ciencias como la psicología, sociología, ciencias sociales entre otras, pues a través de ella se estudia a los individuos y a los grupos dentro de las organizaciones. En este sentido Consuelo et al. (2018) menciona que es difícil establecer una separación entre el comportamiento de las personas y el de las organizaciones. Esta herramienta permite analiza e investiga cómo se relacionan individuos dentro de la estructura de la organización, esto brinda información que puede ser utilizado para medir la eficacia dentro de las áreas de trabajo. En este sentido Morejón (2018) menciona que "el comportamiento organizacional desde el punto de partida se presenta con (los empleados, la rotación de puestos, el rendimiento de los subordinados, la productividad y la gerencia)".

Para poder realizar estudios basados en el comportamiento organizacional es necesario considerar los valores que poseen los trabajadores pues estos son la base fundamental para poder entender la motivación y las actitudes de las personas, ya que estos aspectos son los 
que intervienen en la manera como las personas perciben el mundo y su alrededor, así como también la forma cómo interactúan con el resto de los individuos (Morejón, 2018).

A través de diversos métodos se puede evaluar el comportamiento organizacional dentro de la organización y esta incluye lo mostrado en la tabla 1.

\section{Tabla 1}

Métodos de investigación del comportamiento organizacional

\begin{tabular}{ll}
\hline \multicolumn{1}{c}{ Método } & \multicolumn{1}{c}{ Concepto } \\
\hline \multirow{3}{*}{ Encuestas } & En formas de entrevistas, cuestionarios o pruebas son las formas como \\
& se puede medir el nivel de satisfacción con el trabajo de un equipo o el \\
& clima organizacional \\
\hline
\end{tabular}

Este método permite estudiar documentos que existen dentro de la

Recopilación de Información organización y regulan las actividades de los empleados o grupos de Fija trabajadores (código corporativo, estatutos, descripciones y regulaciones de trabajo, así como los contratos)

Se realiza a través del estudio del estado y la situación del lugar de

Observación trabajo, la apariencia de los empleados en función de los requisitos de la cultura organizacional.

Experimentos Se realizan experimentos de forma natural o de laboratorio-

Fuente: CEUPE Magazine (2021)

\section{Modelos del comportamiento organizacional}

Como ya se ha mencionado, los trabajadores se comportan de diferentes maneras dentro de la empresa, cada persona decide cómo será su conducta, y su manera de aceptar o no la filosofía de la organización.

En este sentido CEUPE Magazine (2021), menciona que según la combinación de los aspectos antes mencionados se pueden diferenciar cuatro modelos de comportamiento organizacional y se muestran en la tabla 2

\section{Tabla 2}

Modelos de comportamiento organizacional

\begin{tabular}{ll}
\hline \multicolumn{1}{c}{ Modelo } & \multicolumn{1}{c}{ Concepto } \\
\hline Miembro disciplinado y & Acepta plenamente todas las normas de comportamiento y los \\
dedicado de la organización & valores establecidos.
\end{tabular}




\section{Tabla 2}

Modelos de comportamiento organizacional (continuación)

\begin{tabular}{ll}
\hline \multicolumn{1}{c}{ Modelo } & \multicolumn{1}{c}{ Concepto } \\
\hline No acepta valores de la organización, pero intenta estructurar su \\
comportamiento completamente de acuerdo con las formas y \\
normas de comportamiento adoptadas en la compañía. Hace todo \\
según las reglas, pero no es considerado un empleado confiable. \\
\hline Acepta los objetivos de la organización, pero no acepta las normas \\
de comportamiento y tradiciones que se han desarrollado en ella. \\
Este tipo de empleado puede generar dificultades en las relaciones \\
tanto con la gerencia como con los compañeros. \\
\hline Es un individuo que no acepta valores o normas de \\
comportamiento. Este empleado es un rebelde, abierto que \\
constantemente entra en conflicto con el medio ambiente y crea \\
situaciones de conflicto. En ocasiones generan problemas que \\
dificultan el desarrollo normal de las actividades dentro de la \\
organización.
\end{tabular}

Fuente: CEUPE Magazine (2021)

Es importante que los directivos tengan en cuenta y conozcan las personalidades de sus trabajadores de manera que puedan guiarlos, y alinearlos acorde a las normativas internas de la organización, pues esto es parte del éxito empresarial (Campos et al., 2016). En el mismo orden de ideas, es importante resaltar que el liderazgo y la motivación son elementos fundamentales dentro de la cultura organizacional, pues permite que los trabajadores puedan ser capaces de enfrentar situaciones de cambio dentro de la empresa y puedan cumplir a cabalidad con sus responsabilidades en sus respectivos puestos de trabajo. El líder juega un rol muy importante y de apoyo durante cualquier cambio que surja en la organización, pues tiene la capacidad de desenvolverse y lograr que el resto del grupo pueda seguirlo y seguir el ritmo que este requiera para afrontar las situaciones y evitar el fracaso. Debe ser una fuente de aprendizaje, de conocimientos que le permita a su equipo ser competitivo (Ferrer, 2018). Según Capa et al. (2018) menciona que el liderazgo es necesario en todos los tipos de organización humana, ya sea en las empresas o en cada uno de sus departamentos, de igual manera establece que es necesario conocer el comportamiento de cada trabajador y así tener una idea más clara durante el proceso de liderazgo. 


\section{Situaciones de cambio en las organizaciones}

Para adentrarse al tema de cambio en las organizaciones es preciso conocer el término "cambio" y González et al. (2017), lo describe como un proceso de reposición de una acción o una cosa en relación con otra. Los cambios en su mayoría son rápidos las personas deben aprender a adaptarse y aceptar situaciones de las cuales no están acostumbrados. Actualmente las empresas han sufrido cambios realmente significativos, producto de variables tanto internas como externas que repercuten en gran medida a su normal funcionamiento; Estos cambios van desde aspectos financieros y tecnológicos, así como estructurales, organizacionales y hasta las estrategias y formas de trabajo se han visto afectadas, lo que conlleva a que sus trabajadores deban aceptar nuevos ritmos de trabajo, adaptarse a diferentes exigencias que si no son adecuadamente aceptadas podrían ser sinónimo de fracaso. En la figura 1 se muestran algunos aspectos importantes que son considerados en los cambios organizacionales

\section{Figura 1}

Cambios dentro de la organización

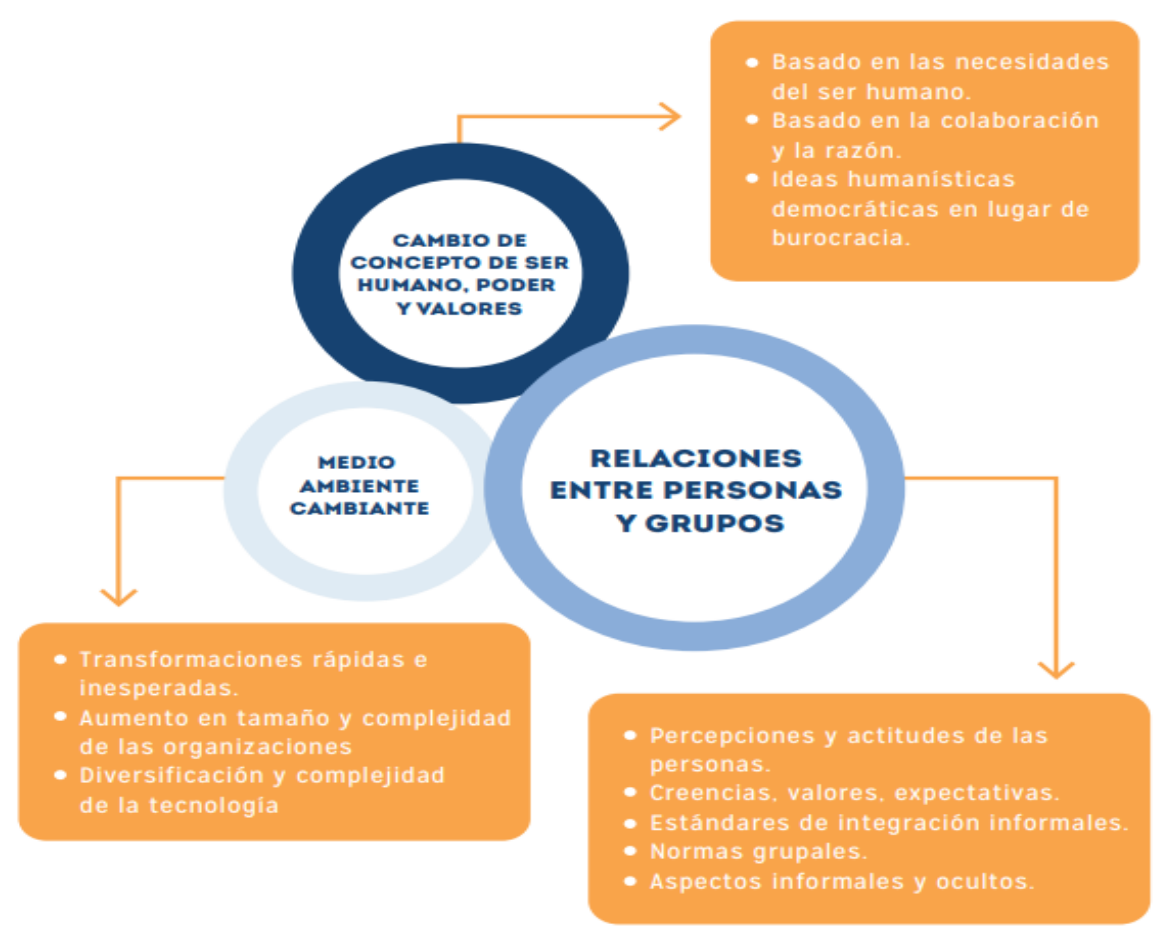

Fuente: Universidad de San Marcos (2016)

Parte de estos cambios, lo conforman la competencia, las exigencias económicas que dan paso a la innovación, pues es un aspecto fundamental para lograr ser una empresa perdurable en el tiempo. 
La mayoría de los cambios que surgen hoy en día en las organizaciones tienen mucho que ver con la implementación de nuevas tecnologías producto de la globalización y los mercados cada vez más exigentes, sobre todo en aquellas empresas con procesos productivos bastantes complejos y de gran envergadura (Rodríguez et al., 2018).

En ocasiones los cambios que deciden los directivos dentro de la organización no son los más adecuados afectando el clima organizacional generando ausentismo, desmotivación baja productividad entre otros, al contrario cuando estos cambios son adecuados y tomados con la mejor actitud por los trabajadores, estos se logran ver beneficiados por ellos, y se nota en los resultados, sin embargo según menciona Cano-Pita (2018), introducir cambios en este caso tecnológicos no garantiza obtener ventajas, ya que esto depende también del conocimiento con que cuenten todos los implicados de cada uno de los procesos de la empresa, de manera que pueda planificarse a detalle cada aspecto para lograr los objetivos planteados.

Hoy en día el capital humano es considerado como el recurso más valioso que tienen las organizaciones, pues son los responsables del funcionamiento de los procesos en cada una de las áreas de las empresas, por lo tanto, se les debe motivar y formar, de manera que mantengan ese compromiso organizacional con la empresa (Prieto et al., 2018)

Es importante mencionar que dentro de los cambios organizacionales no se puede excluir el ambiente multicultural que existe actualmente, que influye en cierta medida en la manera como se desenvuelve la fuerza laboral.

Técnicas que pueden ser utilizadas para administrar de forma adecuada el cambio en las organizaciones

Debido a los vertiginosos cambios mundiales, las organizaciones deben poseer culturas que sean flexibles, lo que permite que sus colaboradores puedan adaptarse y sean capaces de seguir adelante en la Figura 2 se muestra el proceso de cambio que puede surgir dentro de las empresas. 


\section{Figura 2}

Proceso de Adopción del cambio

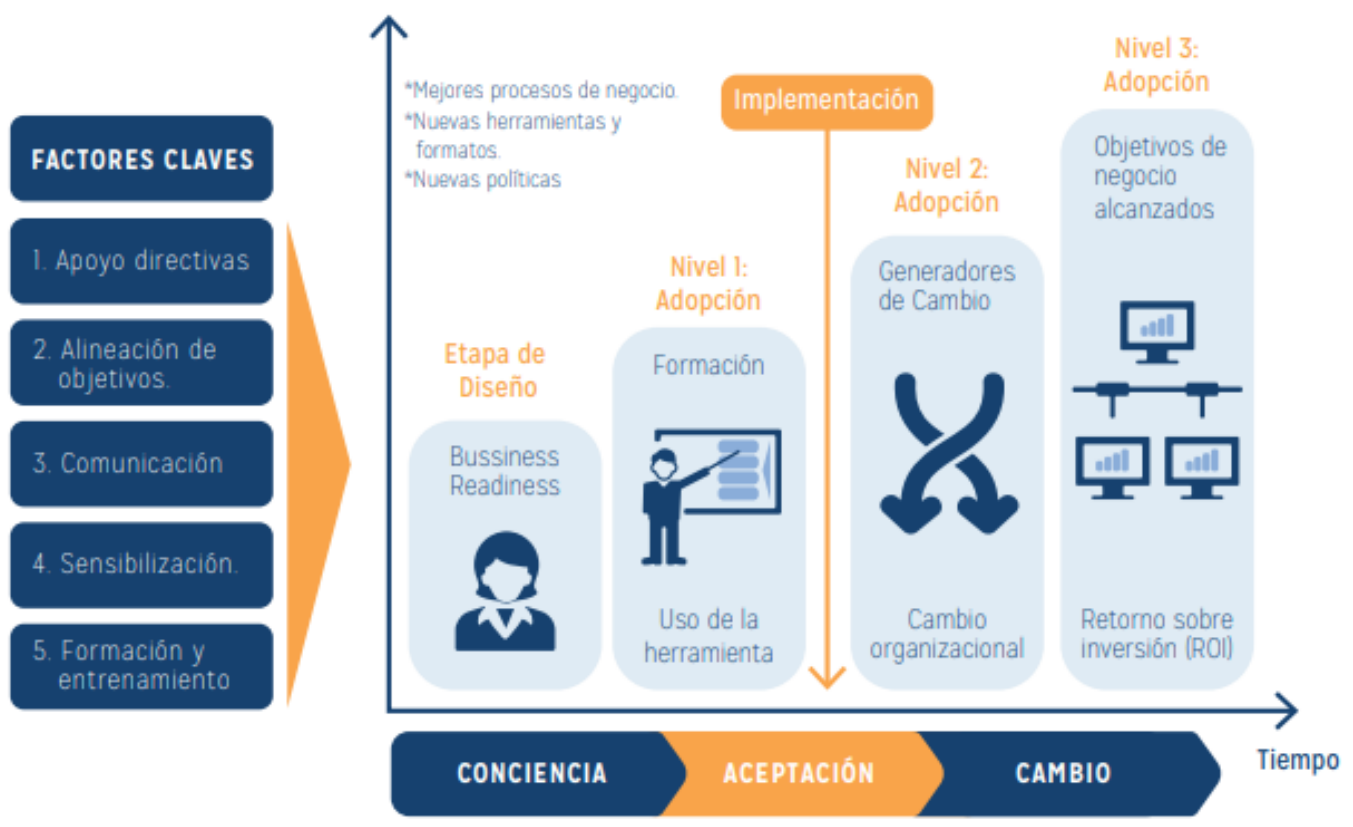

Fuente: Universidad de San Marcos (2016)

A pesar de todos los aspectos positivos presentes en los cambios organizaciones también surgen algunos impedimentos que pueden evitar que la organización progrese de forma adecuada frente a estos cambios. Una falta de visión hacia el futuro de la empresa o hacia donde se desea posicionar la empresa, así como una falta de juicio o la falta de capacidad para analizar las situaciones de forma adecuada puede perjudicar el desenvolvimiento de la empresa.

De igual manera las actitudes defensivas de los empleados y la poca aceptación a los cambios por antiguas maneras de pensar causan también que estos cambios no puedan ser adaptados o aplicados dentro de la empresa. De allí proviene el hecho de mantener una cultura organizacional armoniosa, con respecto y a la vez con un buen liderazgo que permita establecer normas y estrategias que se adapten al grupo de trabajo y que estos a su vez sean capaces de responder con actitudes adecuadas frente a los cambios.

\section{Conclusiones}

- Las organizaciones deben tener muy claras las metas y objetivos trazados, con el propósito de definir estrategias que se adecúen para el logro de estas, así como también conocer las conductas de cada uno de sus trabajadores, de manera que la 
estructura organizacional no se vea afectada por posibles cambios que puedan surgir dentro de la empresa.

- Asimismo, es importante que los directivos tomen en consideración la motivación y el liderazgo como aspectos fundamentales para lograr que la cultura organizacional sea armónica, y en concordancia con los valores, misión y visión de la organización, donde fluya una comunicación entre todos los niveles de la estructura organizacional.

- Los cambios son parte de la evolución de las organizaciones, por lo que la preparación, el aprendizaje y el mejoramiento profesional son herramientas que sirven para cubrir las necesidades que se tengan en las áreas de trabajo, y sean capaces de atravesar estos cambios de forma positiva, que sean sinónimo de progreso y beneficios para todos.

\section{Referencias Bibliográficas}

Armijo, M., \& Portalanza, C. (2017). Diagnóstico de la calidad de vida laboral, engagement y burnout en trabajadores de una institución de educación superior del Ecuador. INNOVA Research Journal 2(12), 153-165.

Campos, H., Espinoza, W., Espinoza, R., \& Zambrano, M. (marzo de 2016). El comportamiento organizacional y su influencia en la calidad y productividad de la organización. Recuperado el 28 de diciembre de 2021, de Revista Caribeña de Ciencias Sociales: https://www.eumed.net/rev/caribe/2016/03/calidad.html

Cano-Pita, G. (2018). Las TICs en las empresas: Evolución de la tecnología y cambio estructural en las organizaciones. Dominio de las Ciencias 4(1), 499-510.

Capa, L., Benítez, R., \& Capa, X. (2018). El liderazgo como fuente de ventaja competitiva para las organizaciones. Universidad y Sociedad 10(2), 273-284.

CEUPE Magazine. (2021). Los 4 modelos de comportamiento organizacional.

Consuelo-Bravo, C., Samentero-Bon, I., Gómez-Figueroa, O., \& Falcón, O. (2018). Procedimiento para el estudio del comportamiento organizacional. Ingeniería Industrial XXXIV (1), 92-100.

Félix, R., García, C., Espinoza, K., Mercado, S., \& Ochoa, E. (2018). Compromiso Organizacional en trabajadores de pequeñas y medianas empresas. CULCYT. Cultura Científica y Tecnológica 15(66), 20-28.

Ferrer, R. (2018). El comportamiento organizacional y su relación en los procesos de innovación tecnológica. ACADEMO. Revista de Investigación en Ciencias Sociales y Humanidades 5(2), 169-178. 
González, J., Rodríguez, M., \& González, O. (2017). Cambio Organizacional en las mediana y grandes empresas del Valle de Sugamuxi. Dimensión Empresarial 5(1), 203-216.

Grajales, T. (2000). Tipos de Investigación. Investipos, 1-4.

Molina-Germán, J., Pérez-Melo, A., \& Lizárraga-Salazar, G. (2019). Estudio de cultura organizacional y satisfacción laboral en empresas comerciales. Ingenio y Conciencia Boletín Científico de la Escuela Superior de Sd. Sahagún 11, 36-43.

Morejón, M. (2018). Comportamiento organizacional: Análisis a partir de su aplicación en la Administración Pública. Revista Enfoques: Ciencia Política y Administración Pública 16(29), 1-16.

Prieto, R., Estrada, H., Palacios, A., \& Paz, A. (2018). Factores del cambio organizacional. Claves del éxito en la gestión de empresas del sector petrolero. Revista de Ciencias Sociales (RCS) 24 (1), 85-100.

Rodríguez, H., Montilla, A., \& Quijije, P. (2018). Clima y Cultura Organizacional y su Relación con el Cmbio Gerencial de Organizaciones Tradicionales a Organizaciones Inteligentes. Revista Ciencias Sociales y Económicas 2(1), 130149.

Universidad de San Marcos. (2016). Cambio y comportamiento organizacional. Universidad de San Marcos.

\section{【 Ciencia}


El artículo que se publica es de exclusiva responsabilidad de los autores y no necesariamente reflejan el pensamiento de la Revista Alfa Publicaciones.

\section{Ciencia}

El artículo queda en propiedad de la revista y, por tanto, su publicación parcial y/o total en otro medio tiene que ser autorizado por el director de la Revista Alfa Publicaciones.
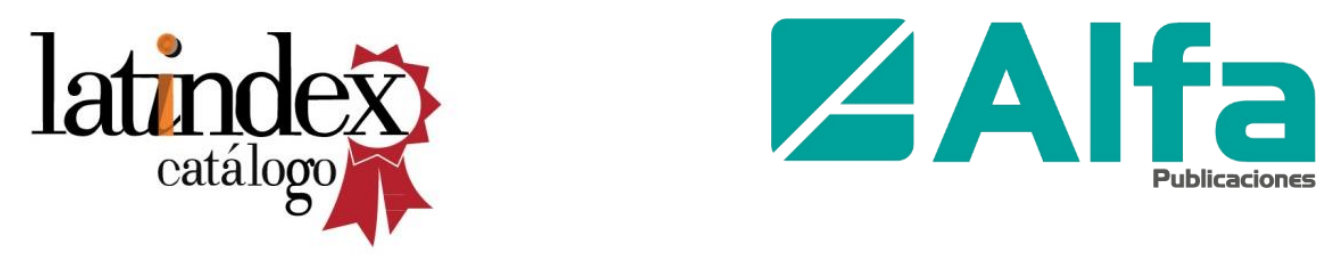

\section{Indexaciones}

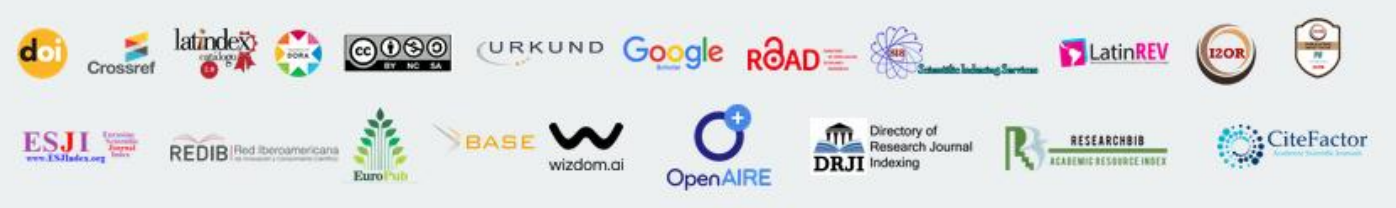

